\title{
LETTER
}

\section{Malignant hyperthermia in the intensive care setting}

\author{
Frank Schuster*, Susanne Moegele, Stephan Johannsen and Norbert Roewer
}

Malignant hyperthermia $(\mathrm{MH})$ is a life-threatening, inherited disorder of skeletal muscle calcium homeostasis. In susceptible individuals, exposure to trigger agents such as volatile anesthetics and depolarizing muscle relaxants, typically administered to induce or maintain general anesthesia, may lead to a hypermetabolic muscular syndrome [1]. Recently, an anesthetic conserving device (AnaConDa'; Sedana Medical AB, Geretsried, Germany) was introduced for sedation of intensive care patients using volatile anesthetics, associated with shorter wake-up and extubation time, reduced duration of mechanical ventilation and earlier hospital discharge [2].

After obtaining oral and written consent, we report the case of a 59-year-old male patient, who was hospitalized due to persistent lumbalgia. On the second day of the patient's hospital stay, increasing dyspnea and tachypnea required admission to the ICU and tracheal intubation. Sedation was maintained by propofol and sufentanil infusion. A chest radiography revealed bilateral pulmonary infiltration, suggestive of influenza pneumonia. Moxifloxacin, piperacillin/tazobactam and oseltamivir were started immediately. After 4 days on mechanical ventilation, sedation needed to be escalated and an anesthetic conserving device for additional sevoflurane sedation was installed. Suddenly, after 5 hours of sevoflurane administration, hemodynamic instability occurred. The arterial blood pressure rapidly decreased to $78 / 44 \mathrm{mmHg}$, while the heart rate remained constant around 110 beats/minute. Arterial blood gas analysis revealed severe acidosis $(\mathrm{pH}$, 7.17; carbon dioxide partial pressure, $70.4 \mathrm{mmHg}$; oxygen partial pressure, $104 \mathrm{mmHg}$ (fraction of inspired oxygen, $50 \%$ ); base excess, 9.8 ; lactate, $0.6 \mathrm{mmol} / \mathrm{l})$, and a rapid increase of body temperature from 39.6 to $40.7^{\circ} \mathrm{C}$ within 30 minutes was noticed. Creatine kinase and myoglobin levels were significantly increased to maximum levels of 3,455 U/l and 4,197 $\mu \mathrm{g} / \mathrm{l}$, respectively, due to acute rhabdomyolysis. Since $\mathrm{MH}$ was suspected, sevoflurane

\footnotetext{
* Correspondence: schuster f@ukw.de

Department of Anesthesia and Critical Care, University of Wuerzburg,

Oberduerrbacher Straße 6, D-97080 Wuerzburg, Germany
}

application was discontinued, and dantrolene (225 mg) was administered intravenously, resulting in gradual improvement of the hemodynamic and metabolic situation. The further course was unremarkable. The subsequent ICU stay remained uneventful and the patient was extubated on day 17 without neurological deficits. Nine months after the suspected MH event, the patient underwent muscle biopsy and in vitro contracture testing according to the guidelines of the European $\mathrm{MH}$ group. The patient's MH susceptibility was thus confirmed, by significant contractures at the defined thresholds after halothane and caffeine exposure [3].

The presented case underlines that $\mathrm{MH}$ is an important differential diagnosis in intensive care patients with hemodynamic and metabolic breakdown if volatile anesthetics are used for sedation. Hence, every ICU physician must be aware of the symptoms of $\mathrm{MH}$ to rapidly initiate sufficient treatment.

\section{Abbreviations \\ $\mathrm{MH}$ : Malignant hyperthermia.}

\section{Competing interests}

The authors declare that they have no competing interests.

\section{Authors' contributions}

FS accompanied the data acquisition, collected and analyzed the data and drafted the manuscript. SM collected the data and performed the muscle biopsy. SJ collected data. NR participated in the design of the study. All authors read and approved the final manuscript.

\section{Acknowledgements}

Written informed consent was obtained from the patient for publication of his individual details in this manuscript. The consent form is held in the patients' clinical notes and is available for review by the Editor-in-Chief. Chief technician Judith Skirde (Malignant Hyperthermia Laboratory, University of Wuerzburg, Germany) contributed essential advice and technical assistance throughout the in vitro contracture test. The investigation was performed at the Department of Anesthesia and Critical Care at the University of Wuerzburg, Germany.

Published: 26 Feb 2014

\section{References}

1. Schuster F, Müller-Reible CR: Malignant hyperthermia - diagnostics, treatment and anaesthetic management. Anasthesiol Intensivmed Notfallmed Schmerzther 2009, 44:758-763. 
2. Misra S, Koshy T: A review of the practice of sedation with inhalational anaesthetics in the intensive care unit with the AnaConDa ${ }^{\oplus}$ device. Indian J Anaesth 2012, 56:518-523.

3. European Malignant Hyperpyrexia Group: A protocol for the investigation of malignant hyperpyrexia (MH) susceptibility. Br J Anaesth 1984, 56:1267-1269.

$10.1186 / \mathrm{cc} 13744$

Cite this article as: Schuster et al: Malignant hyperthermia in the intensive care setting. Critical Care 2014, 18:411 